J3eA - Vol. 3 - 6 (2004).

DOI : 10.1051/bib-j3ea:2004006

\title{
Magnétisme microscopique à l'échelle des domaines magnétiques dans les matériaux ferromagnétiques doux
}

\author{
F. Alves ** et R. Barrué ** (ENS Cachan) \\ Mis en ligne le 03/09/2004.
}

\begin{abstract}
Résumé
La visualisation des domaines magnétiques de Weiss constitue un outil d'analyse intéressant et utile, aussi bien du point de la compréhension des phénomènes de base, que du point de la caractérisation des matériaux fonctionnels. Elle permet, dans les matériaux cristallins, de vérifier si les textures de GOSS sont bien dans le plan de laminage des tôles fer-silicium. Dans les alliages amorphes, elle apporte une aide à l'analyse de l'efficacité des traitements thermiques (relaxation des contraintes internes, induction d'une anisotropie magnétique) ; par ailleurs, la mobilité des parois et la largeur des domaines apportent des informations sur la perméabilité et les pertes électromagnétiques. Un dispositif basé sur l'effet Kerr magnéto-optique longitudinal est décrit dans cet article. Un certain nombre de structures en domaines dans différents matériaux ferromagnétiques sont présentées. Grâce à différentes séquences vidéo numériques originales, nous montrons ce qu'est l'hystérèse magnétique à l'échelle des domaines.
\end{abstract}

Mots-clés : domaines magnétiques, vidéo numérique, hystérèse, couplage magnétiquemécanique, tôles fer-silicium, matériaux amorphes, alliages nanocristallins.

(C) EDP Sciences, 2004.

Niveau de connaissances requis. Notions d'électromagnétisme.

Niveau des étudiants. Deuxième cycle Génie électrique/EEA, physique...

* F. Alves ${ }^{1}$ est maître de conférences (HdR) à l'École Normale Supérieure de Cachan (ENSC), détaché depuis 1995 au département de Génie Électrique de l'IUFM de Créteil. Il prépare les étudiants aux concours de recrutement d'enseignants du second degré en lycées techniques et professionnels (CAPET et CAPLP). Son activité de recherche, menée au SATIE, est centrée sur deux thèmes : l'étude de matériaux magnétiques nanostructurés et leur apport en électronique de puissance, le développement, dans un cadre européen, de microcapteurs magnétiques en vue d'applications dans le domaine du contrôle non destructif et du biomédical.

a e-mail : alves@satie.ens-cachan.fr

** R. Barrué ${ }^{1}$ est professeur des universités à l'ENSC et est responsable du pôle de compétences « Matériaux magnétiques » de SATIE.

e-mail : barrue@satie.ens-cachan.fr

${ }^{1}$ SATIE UMR CNRS 8029, ENS Cachan, 61 avenue du Président Wilson, F-94235 Cachan, France.

Article available at http://www.j3ea.org or http://dx.doi.org/10.1051/bib-j3ea:2004006 


\section{Matériaux ferromagnétiques et structure en domaines}

Tous les matériaux présentant un magnétisme important, donc utilisables industriellement, comme les ferro- et les ferrimagnétiques, sont des matériaux hétérogènes d'un point de vue de la structure magnétique. Ils sont saturés localement; par contre, à l'échelle macroscopique, en l'absence de champ magnétique extérieur, ils peuvent avoir :

- soit un comportement saturé, c'est le cas des matériaux durs appelés aussi aimants permanents, dans lesquels tout est figé du point de vue des structures en domaines ;

- soit un comportement plus modéré que l'on rencontre dans les matériaux magnétiques dits doux. Ces derniers, en l'absence de champ extérieur, présentent pratiquement une aimantation macroscopique nulle.

Il importe donc de bien distinguer le vecteur aimantation macroscopique (échelle de l'utilisateur), du vecteur aimantation microscopique (échelle atomique). Le premier de ces deux vecteurs est une moyenne spatiale du second vecteur ; c'est une constante vectorielle au sein de chaque domaine (hors parois), en régime quasistatique. Il est clair qu'en tout point du matériau, le vecteur aimantation microscopique, dont la direction peut changer, a partout le même module et que son module a pour valeur l'aimantation à saturation.

L'idée des domaines n'est pas récente, celui qui l'a mise en conjecture dans son travail de thèse en 1907 est Pierre Weiss. Il a montré qu'un échantillon de taille macroscopique, afin de diminuer son énergie globale, se subdivise en de nombreuses régions aimantées à saturation (domaines) séparées par des frontières (parois de Bloch ou parois de Néel). Dans ce cas, l'aimantation globale de la substance n'est égale à l'aimantation spontanée que lorsque celui-ci est saturé. C'est donc une idée qui a été avancée, il y a longtemps, néanmoins, la visualisation de telles structures et les interprétations que l'on peut en faire sont loin d'être un sujet de recherche épuisé.

Les processus d'aimantation sont dominés par les changements topologiques des structures en domaines (déplacements de parois et rotations d'aimantation) sous l'action d'un champ magnétique extérieur. Les pertes électromagnétiques dissipées correspondent à des pertes Joule (générées par des densités de courants de Foucault, localisées autour des parois en mouvement, ou réparties dans l'ensemble d'un domaine en rotation) et/ou à des pertes par relaxation de spins (là encore, localisées au sein des parois en mouvement, ou réparties dans l'ensemble d'un domaine en rotation).

Pour visualiser ces structures en domaines magnétiques qui guident tous les processus d'aimantation et de génération de pertes dans les matériaux magnétiques doux, diverses méthodes d'investigation existent. Les premières ont été développées à partir de poudres très fines par Bitter en 1931. En 1935, Landau et Lifshitz ont relié l'existence des domaines de Weiss aux figures de Bitter. Ils ont montré que ce n'était là qu'une conséquence naturelle de la minimisation des diverses énergies mises en jeu dans les corps ferromagnétiques :

- l'énergie magnétostatique (liée aux champs démagnétisants internes qui tendent à minimiser le parcours des lignes de champ dans l'air par la création de nouveaux domaines);

- l'énergie de paroi (stockée dans la paroi, elle tend à équilibrer la diminution de l'énergie magnétostatique);

- l'énergie magnétocristalline (liée au fait qu'il existe des axes de facile aimantation dans le réseau cristallin et que la rotation des moments magnétiques demande une mise en jeu importante d'énergie) ;

- l'énergie d'échange (qui résulte de l'interaction des moments et qui passe par un minimum lorsqu'ils sont alignés)

- et l'énergie magnéto-élastique (qui résulte de l'orientation préférentielle de l'aimantation lorsque le cristal est sous contrainte mécanique).

C'est sous l'action de ces diverses énergies que s'établit la structure en domaines magnétiques.

Vers les années 50, sont apparues des techniques de visualisation plus fines avec l'utilisation des ferro-fluides, puis le renfort du polissage électrolytique. À partir des années 60 , sont mises au point des méthodes optiques plus lourdes et plus sophistiquées ; il s'agit de la microscopie électronique à balayage (MEB) et des méthodes basées sur les effets magnéto-optiques (effet Faraday et effet Kerr), autorisant la visualisation dynamique (sous 
excitation magnétique extérieure variable). Notons toutefois que les techniques MEB d'investigation nécessitent un appareillage lourd, coûteux et permettant difficilement d'effectuer certaines manipulations comme des tractions ou des variations de température in situ.

Nous allons maintenant décrire brièvement les différents termes d'énergie mentionnés plus haut.

\section{L'énergie magnétostatique $\mathbf{E}_{\mathrm{ms}}$}

Quand on aimante un matériau ferromagnétique, l'énergie magnétostatique dans l'échantillon à la saturation est donnée par:

$$
E_{i m s}=\mu_{0} \int_{0}^{M s}\left(\vec{H}_{e x t}+\vec{H}_{d}\right) \cdot d \vec{M}=\mu_{0} \int_{0}^{M s}\left(\vec{H}_{e x t}-N_{d} \vec{M}\right) \cdot d \vec{M}
$$<smiles>C=[Ru]CC</smiles>

Hd

où représente le champ extérieur appliqué, le champ démagnétisant, $\overrightarrow{\mathrm{M}}_{\text {l'aimantation, } \mathrm{N}_{\mathrm{d}} \text { le facteur }}$ démagnétisant et $\mathrm{M}_{\mathrm{s}}$ l'aimantation à saturation. Le champ démagnétisant représente la réaction du matériau à l'application d'un champ extérieur. Dans le cas d'un échantillon allongé, celui-ci s'aimante plus facilement le long de son grand axe que dans une direction perpendiculaire. Cela provient de la valeur du champ démagnétisant, qui est différente suivant les directions. Pour une géométrie de type ruban, le champ démagnétisant peut être exprimé par la formule suivante :

$$
\overrightarrow{\mathrm{H}}_{\mathrm{d}}=-\mathrm{N}_{/ /} \cdot \overrightarrow{\mathrm{M}}_{/ /}
$$

où $\mathrm{N}_{/ /}$représente le facteur démagnétisant dans la direction longitudinale, la composante longitudinale de l'aimantation.

On en déduit que :

$$
E_{m s}=\mu_{0} \vec{H}_{e x t}, \vec{M}_{s}-\mu_{0} \int_{0}^{\mathrm{Ms}}\left(\mathrm{N}_{/ /} \overrightarrow{\mathrm{M}}_{/ /}\right), \mathrm{d} \overrightarrow{\mathrm{M}}_{/ /}
$$

d'où finalement :

$$
\mathrm{E}_{\mathrm{ms}}=\mu_{0} \overrightarrow{\mathrm{H}}_{\mathrm{ext}}=\overrightarrow{\mathrm{M}}_{\mathrm{s}}-\mu_{0} \mathrm{~N}_{/} / \frac{\mathrm{M}_{\mathrm{s}}^{2}}{2}
$$

Cette dernière expression comporte deux termes : l'un d'interaction avec le champ qui dépend seulement des propriétés de la matière, l'autre qui dépend aussi de la forme. Il peut ainsi apparaître une anisotropie de forme importante, particulièrement pour les corps divisés ou comportant plusieurs phases (fabrication des aimants permanents).

\section{L'énergie magnéto-élastique}

\section{$E_{\sigma}$}

L'application d'une contrainte provoque une déformation du réseau atomique qui va induire une énergie d'anisotropie. Dans le cas d'une contrainte située dans le domaine élastique et pour un matériau considéré comme isotrope, cette énergie, notée $E_{\sigma}$, est donnée par :

$$
E_{\sigma}=-\frac{3}{2} \cdot \lambda_{\mathrm{g}}, \sigma \cdot \cos ^{2} \theta
$$




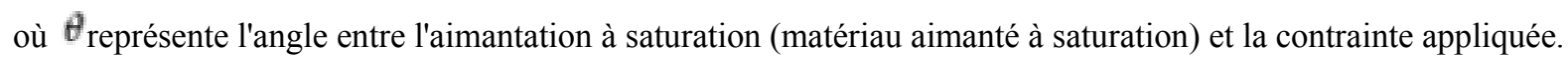

$\lambda_{\mathrm{s}}$

, appelé coefficient de magnétostriction à saturation, représente l'allongement relatif maximal du matériau lorsque que celui-ci est sous l'effet d'une aimantation à saturation.

Pour mieux visualiser les effets d'une contrainte sur les propriétés magnétiques d'un matériau, on peut s'intéresser aux courbes de première aimantation, relevées lorsqu'il est soumis à différentes contraintes. L'aire

\section{$\mathrm{BHJ}$}

comprise entre l'axe des polarisations magnétiques (dans les matériaux doux, on peut écrire que ) et la courbe de première aimantation représente l'énergie d'anisotropie de l'échantillon. Par conséquent, l'aire comprise entre la courbe de première aimantation du matériau soumis à la contrainte $\sigma$ et la courbe de première aimantation du même matériau, libre de toute contrainte, représente l'énergie d'anisotropie induite par la variation de la contrainte $\sigma$.

$\mathrm{J}(\mathrm{T})$

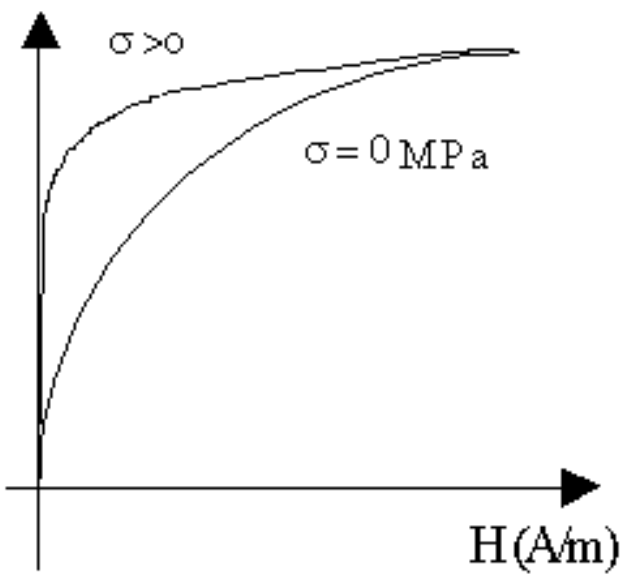

(a) Energie in cuite négative magnétostriction positive
$\mathrm{J}(\mathrm{T})$

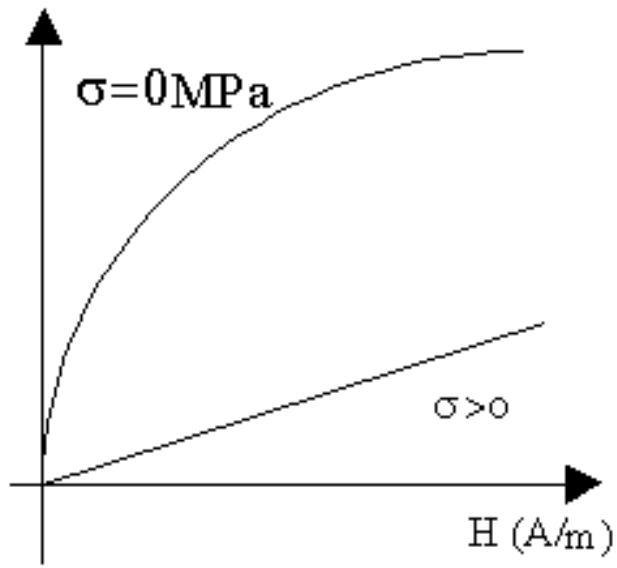

(b) Energie induite positive magnétostriction négative

Fig. 1. Effet d'une contrainte sur la courbe de première aimantation d'un matériau suivant la signe de son coefficient de magnétostriction à saturation.

Pour un matériau à magnétostriction positive, on observe qu'une traction ( ) induit une énergie négative. Dans ce cas, la courbe de première aimantation se redresse. Inversement, pour un matériau à magnétostriction négative, une traction induit une énergie positive (Fig. 1). La courbe de première aimantation se couche.

\section{L'énergie d'échange $\mathbf{E}_{\text {ech }}$}

L'énergie d'échange est une énergie résultant des interactions entre atomes, via leur spin total.

Considérons deux atomes (i) et (j), l'expression de l'énergie d'échange est donnée par :

$$
E_{\text {ech }}=-2 J_{e c b_{i, j}}\left(\vec{S}_{i} \cdot \vec{S}_{j}\right)
$$

Le signe de l'intégrale d'échange $\mathrm{J}_{\text {echi,j }}$ définit le magnétisme du matériau. Dans le cas du ferromagnétisme, $\mathrm{J}_{\text {echi,j }}$

$$
\overrightarrow{\mathrm{S}}_{\mathrm{i}} \overrightarrow{\mathrm{S}}_{\mathrm{j}}
$$

est positif ce qui impose à et (moments de spin) d'être alignés dans le même sens. Cela permet de 
minimiser l'énergie d'échange, afin de tendre vers la structure magnétique la plus stable (à l'intérieur d'un domaine magnétique, tous les moments magnétiques sont orientés dans le même sens).

\section{L'énergie magnétocristalline $\mathbf{E}_{\mathrm{K} 1}$}

Dans un matériau ferromagnétique cristallin, le vecteur aimantation ne peut pas prendre n'importe quelle direction. Certaines directions cristallographiques, appelées directions de facile aimantation, sont privilégiées.

Le travail d'aimantation, c'est-à-dire l'énergie à fournir pour amener le matériau de l'état désaimanté à un état aimanté donné, dépendra de l'angle du champ extérieur par rapport aux directions cristallographiques. Ceci permet de dégager une ou plusieurs constantes dites constantes d'anisotropie.

Dans un cristal cubique centré, la densité d'énergie volumique s'écrit :

$$
\mathrm{E}_{\mathrm{I}_{1}}=\mathrm{K}_{1}\left(\alpha_{1}^{2} \alpha_{2}^{2}+\alpha_{1}^{2} \alpha_{3}^{2}+\alpha_{2}^{2} \alpha_{3}^{2}\right)+\mathrm{K}_{2} \alpha_{1}^{2} \alpha_{2}^{2} \alpha_{3}^{2}
$$

où les coefficients $\alpha$ représentent les cosinus directeurs liés aux directions cristallographiques.

Dans la plupart des cas $\mathrm{K}_{2}<\mathrm{K}_{1}$, et par conséquent si $\mathrm{K}_{1}>0$; l'équilibre stable ( $\mathrm{E}_{\mathrm{K} 1}$ minimum) est obtenu pour la direction [100], par contre si $\mathrm{K}_{1}<0$ alors l'équilibre stable est obtenu pour la direction [111] (Fig. 2).

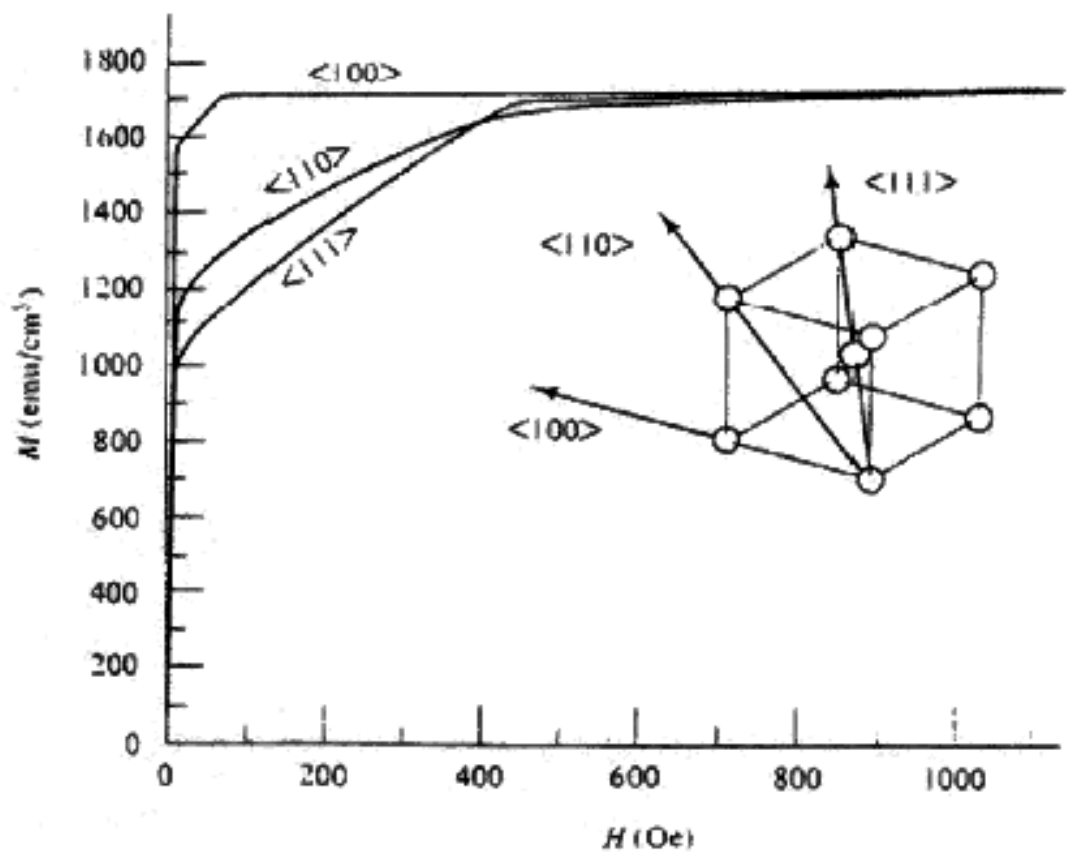

Fig. 2. Courbes d'aimantation selon les trois axes dans le cas d'un monocristal de fer.

\section{L'énergie de paroi $E_{p}$}

Il existe une autre énergie, découlant des précédentes, qui contribue à la configuration de la structure en domaines, c'est l'énergie des parois.

En effet, le passage d'un domaine de direction donnée à un autre domaine de direction différente ne peut pas se faire brutalement (entre deux atomes consécutifs) sans augmenter, localement, le terme d'énergie d'échange d'une quantité importante. Il se forme une zone entre ces deux domaines où les moments de spin des atomes ne sont pas parallèles et assurent une transition angulaire « douce ». Ces zones sont appelées des parois de Bloch (rotation hélicoïdale des spins, donc hors du plan des domaines) ou parois de Néel (rotation des spins dans le plan des domaines). Une modification locale de l'énergie d'échange et de l'énergie d'anisotropie magnétocristalline induit la notion d'épaisseur de la paroi. L'énergie stockée dans la paroi correspond à 
l'augmentation de l'énergie interne nécessaire pour « désaligner » les moments de spin (modification de l'énergie d'échange), et de les éloigner de la direction de facile aimantation (modification de l'énergie d'anisotropie magnétocristalline).

À chaque fois qu'une paroi se crée, $\mathrm{E}_{\mathrm{ms}}$ diminue, mais $\mathrm{E}_{\mathrm{ech}}$ et $\mathrm{E}_{\mathrm{K} 1}$ augmentent. Il se crée donc un équilibre qui limite le nombre de domaines (Fig. 3).
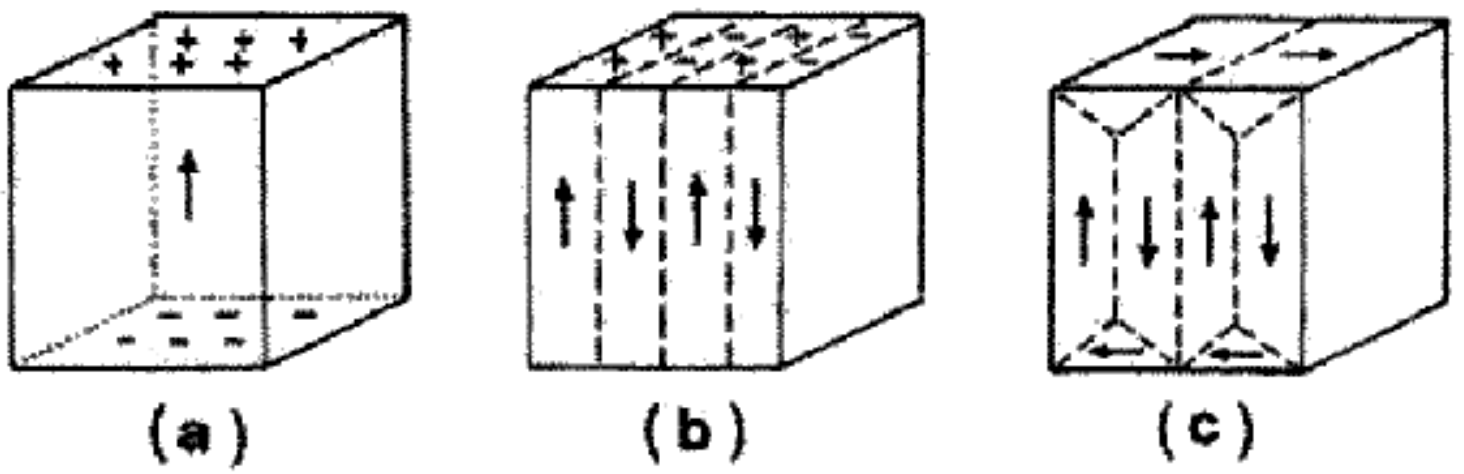

Fig. 3. Exemples de configurations en domaines magnétiques d'un monocristal de fer : (a) aimantation uniforme ; (b) aimantation en domaines antiparallèles ; (c) domaines antiparallèles accompagnés de domaines de fermeture.

La configuration (a) minimise les énergies d'échange et d'anisotropie mais l'énergie magnétostatique est alors importante car les " pôles » sont éloignés les uns des autres (ceci est à prendre en considération par rapport aux autres échantillons de même taille).

Dans le cas de la configuration (b), l'énergie magnétostatique est diminuée, en revanche l'énergie d'échange augmente (pour un échantillon de même taille que le (a)).

Dans la configuration (c), l'introduction de domaines de fermeture supprime les pôles et donc l'énergie magnétostatique. En revanche, si le monocristal est magnétostrictif, les domaines à $90^{\circ}$, qui tendent à se déformer dans la direction de l'aimantation du domaine, font apparaître une énergie magnéto-élastique. Dans ce cas de figure, la largeur des parois résulte de la balance entre l'énergie magnéto-élastique et l'énergie de paroi.

Calcul de l'épaisseur d'une paroi, de quels paramètres dépend-elle?

Considérons la figure ci-dessous (Fig. 4) et calculons le nombre $\mathrm{n}$ d'atomes contenus dans cette paroi dans le cas d'une structure cristalline cubique ( 1 atome par maille).
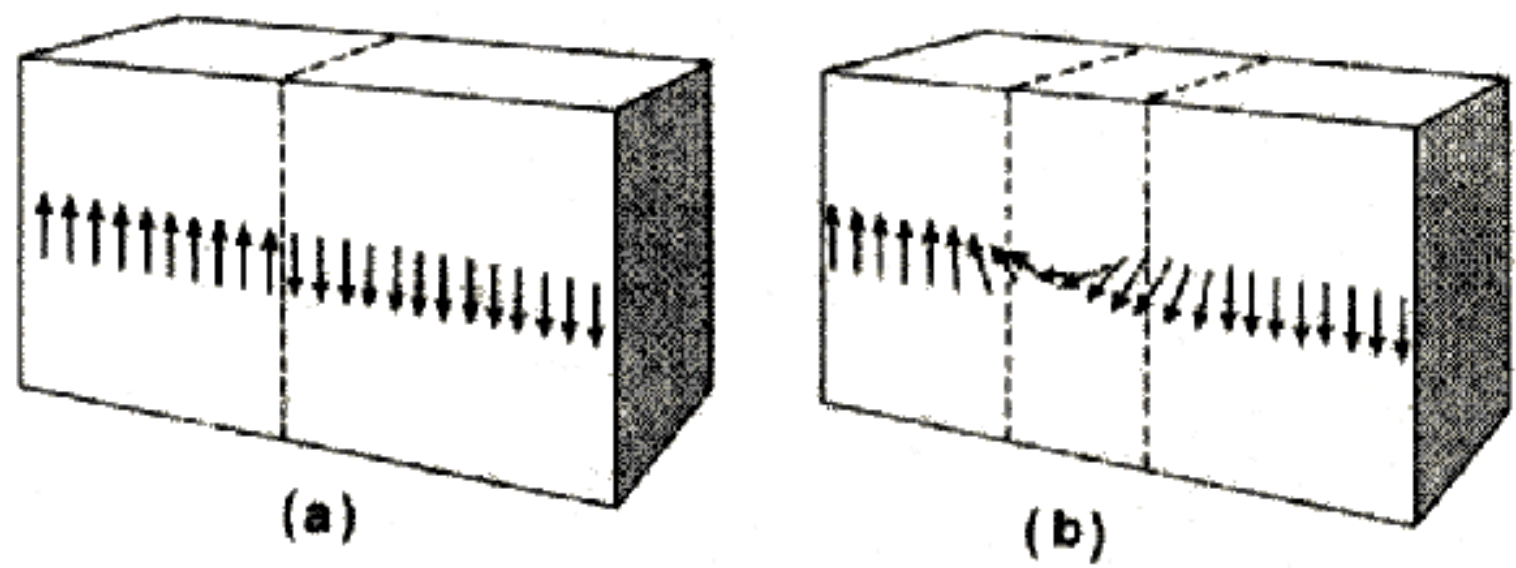

Fig. 4. Transition entre deux domaines à $180^{\circ}$ : (a) la transition est brutale, ce cas n'existe pas ; (b) la transition est progressive, la zone de transition est, ici, une paroi de Bloch. 
Hypothèse de calcul : la rotation des spins dans la paroi est uniforme (n grand) et donc l'angle ${ }^{\Phi_{i j}}$ entre deux $\pi / n$

spins voisins est égal à et très inférieur à 1.

Dans cette paroi, deux énergies coexistent : l'énergie d'échange et l'énergie magnétocristalline.

Energie d'échange stockée dans la paroi

$E_{\mathrm{ech}}=-2 J_{\mathrm{ech}} \mathrm{S}^{2} \sum_{1}^{n} \cos \Phi_{\mathrm{ij}}=-2 J_{\mathrm{ech}} \mathrm{S}^{2} \mathrm{n}\left(1-\frac{\pi^{2}}{2 \mathrm{n}^{2}}\right)$

$\cos \Phi_{i j}$

(résultat du développement limité de

Cette énergie tend à accroître l'épaisseur de la paroi.

Energie magnétocristalline stockée dans la paroi

La rotation des spins dans la paroi hors de la direction de facile aimantation cause un accroissement de l'énergie d'anisotropie magnétocristalline dans la paroi :

$$
\mathrm{E}_{\mathrm{K} 1}=\mathrm{K}_{1} \mathrm{n} \mathrm{a}^{3}
$$

où a représente le paramètre de maille d'un système cubique.

L'énergie totale dans le monocristal est :

$E_{\text {totale }(N \text { atomes }}=-2 J_{e c h} S^{2} n+J_{e c h} S^{2} \frac{\pi^{2}}{n}-2 J_{e c h} S^{2}(N-n)+K_{1} n a^{3}+0=J_{e c h} S^{2} \frac{\pi^{2}}{n}+K_{1} n a^{3}$ (l'énergie magnétocristalline liée aux N-n spins est nulle car ils sont orientés dans une direction de facile aimantation).

La recherche de n passe par la minimisation de l'énergie totale. On obtient alors

$$
\mathrm{n} \equiv \mathrm{S} \pi \sqrt{\frac{\mathrm{J}_{\mathrm{ach}}}{\mathrm{K}_{1} \mathrm{a}^{3}}}
$$

L'épaisseur d de la paroi devient :

$$
\mathrm{d}=n \mathrm{a}=\pi \sqrt{\frac{\mathrm{Jech}_{\mathrm{e}} \mathrm{S}^{2}}{\mathrm{a}_{\mathrm{a}}} \frac{1}{\mathrm{~K}_{1}}}
$$




\section{Visualisation des domaines}

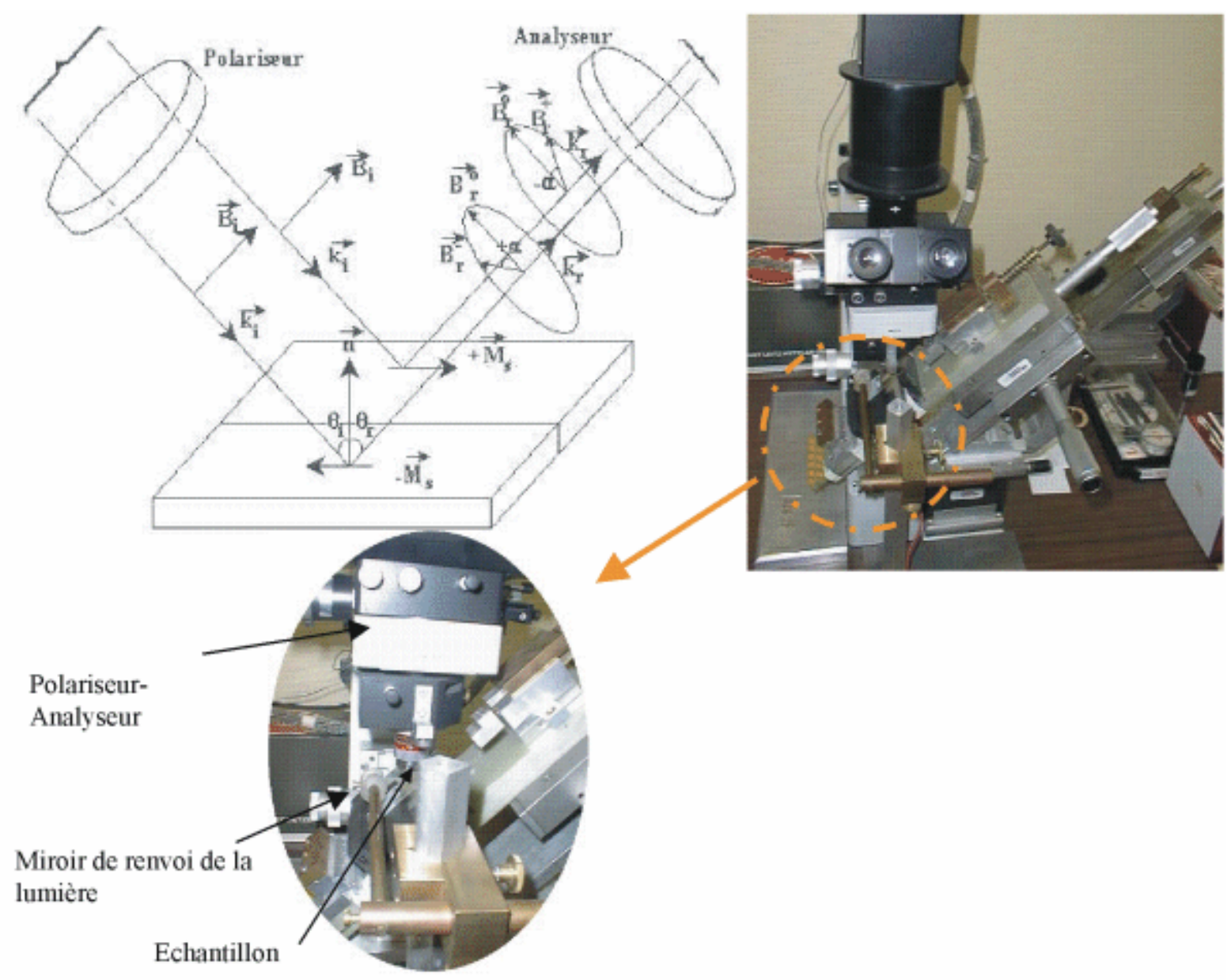

Fig. 5. Principe et dispositif expérimental de l'imagerie par effet Kerr magnéto-optique.

Cette méthode (Fig. 5) est basée sur la modification de la polarisation d'un faisceau lumineux, due à l'interaction avec la matière magnétique dont les propriétés diffèrent d'un domaine à l'autre. Le dispositif expérimental est composé d'un système optique (mise au point de l'effet Kerr) et d'un système d'imagerie comportant une caméra reliée à un dispositif de traitement d'images. Grâce au traitement numérique des images, le système permet de visualiser des domaines qui ne sont pas visibles directement à l'oeil au travers de l'analyseur et évite le polissage des surfaces. Ce traitement d'image comprend la soustraction et le réglage du contraste non magnétique.

\section{Visualisation statique}

Si on considère un faisceau lumineux polarisé, défini par le trièdre EBK, respectivement champ électrique, induction magnétique et vecteur d'onde, et sa réflexion sur un matériau magnétique : l'angle d'incidence est égal à l'angle de réflexion. Si le matériau n'est pas magnétique, il n'y a pas de rotation du plan de la polarisation de la lumière incidente. Au cours de la réflexion de la lumière sur le matériau, se développent, sur une très fine couche, des courants de Foucault à une fréquence très élevée (celle de l'onde électromagnétique qui est de l'ordre de $\left.10^{14}-10^{15} \mathrm{Hertz}\right)$. Ces petits courants de Foucault, qui pénètrent de quelques centièmes de micromètres dans le matériau, sont à l'origine de pertes par réflexion. La rotation de Kerr (c'est son nom) est de l'ordre de 3 minutes dans les matériaux fer-silicium. Un artefac, utilisant un très bon système polariseur-analyseur convenablement croisé, fait apparaître ce qui s'est réfléchi sur certains domaines en noir (absence de lumière) ; pour les autres catégories de domaines, on a 6 minutes d'écart par rapport à ce noir, c'est à dire un peu de lumière, qui, après 
amplification donne une lumière « blanche ». Si on tourne le polariseur de 6 minutes dans l'autre sens, on inverse les blanc-noir. On sait que là il y a des domaines à $180^{\circ}$ mais on est incapable de dire si l'aimantation locale est dirigée vers la droite ou vers la gauche.

\section{Visualisation dynamique}

L'avantage de l'effet Kerr réside dans la possibilité d'observer les processus d'aimantation en régimes statique et dynamique. Il faut utiliser, puisque l'on va hacher la lumière, une source lumineuse plus intense et, en général, un laser est recommandé. On utilise alors la stroboscopie pour observer la dynamique des parois de domaines. Si on désire atteindre des fréquences assez élevées (par exemple jusqu'à dizaines de $\mathrm{kHz}$ ), il faut absolument une synchronisation parfaite des éclairs lumineux et de l'alimentation qui fait varier le champ dans le matériau magnétique ; pour cela on peut utiliser un modulateur acousto-optique. À partir de cette stroboscopie, on peut diminuer artificiellement le déplacement des parois et l'observer jusqu'à des fréquences élevées, typiques de l'électronique de puissance.

\section{Différentes influences sur la structure en domaines}

\subsection{Influence de la température}

Les matériaux amorphes (ou dits aussi verres métalliques, classe de matériaux sans texture cristalline) et leurs dérivés que sont les matériaux nanocristallins sont obtenus par la technique de la trempe d'un alliage en fusion. On peut les fabriquer industriellement soit par la technique de « jet libre » qui consiste à projeter un jet de métal liquide, dont la composition a été déterminée auparavant, sur une roue refroidie tournant à grande vitesse $(30 \mathrm{~m} / \mathrm{s})$. On obtient ainsi des vitesses de refroidissement de l'ordre du million de degrés par seconde. Le deuxième procédé est le « flot planaire ». Là, il s'agit d'une buse assez large permettant de fabriquer des rubans (épaisseur de quelques dizaines de $\mu \mathrm{m}$ ) de grande largeur pouvant atteindre $1 \mathrm{~m}$. À ces vitesses de refroidissement, l'état vitreux peut être obtenu. Il n'y a donc pas de structure cristalline. Des traitements thermiques post-trempe sont nécessaires pour relaxer les contraintes issues du mode d'élaboration et/ou induire un axe de facile aimantation selon l'application industrielle visée. L'électronique de puissance constitue le terrain d'expérimentation le plus propice. La figure 6 montre comment les structures en domaines sont modifiées en fonction de différents recuits thermiques. 


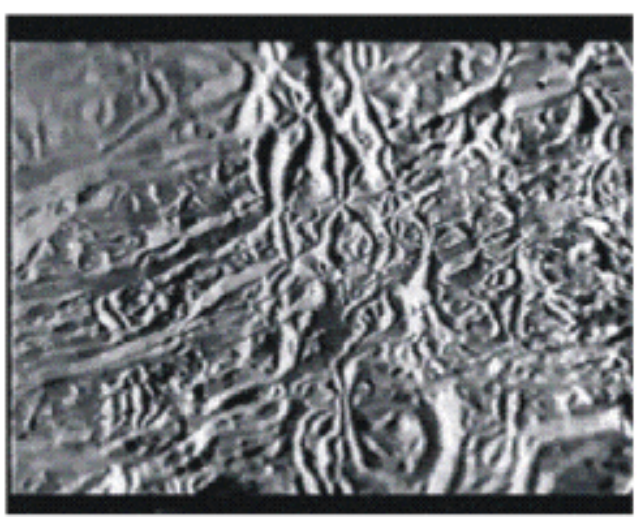

Brut de trempe

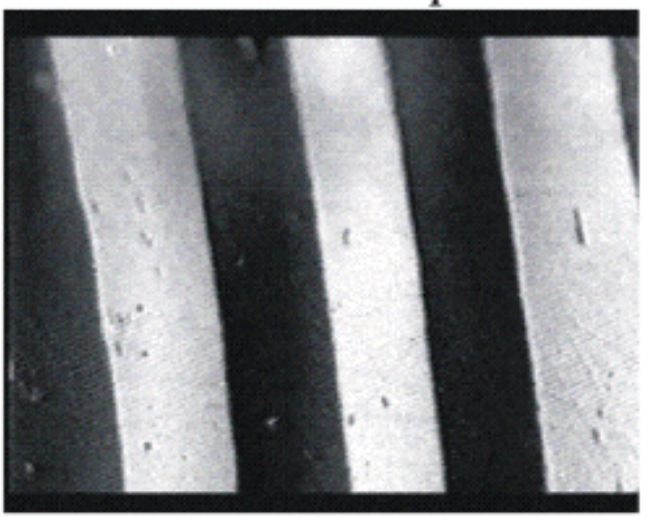

après recuit thermique sous champ longitudinal

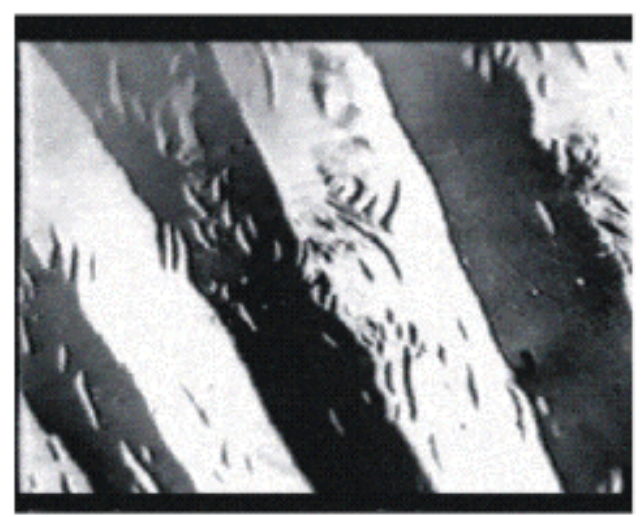

après recuit thermique

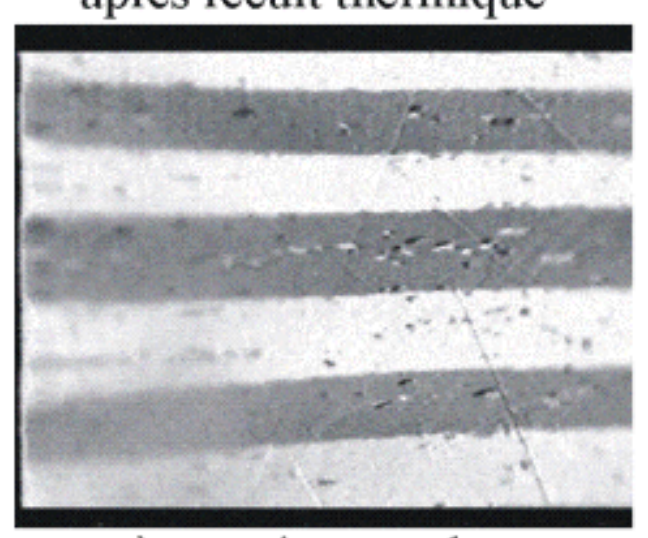

après recuit sous champ

transverse

\section{] Axe longitudinal du ruban}

Fig. 6. Structures en domaines d'un alliage amorphe. Le format des images est de $2 \mathrm{~mm} \times 3 \mathrm{~mm}$.

\subsection{Influence d'une contrainte mécanique}

À l'origine amorphes, les matériaux de composition Fe-B-M $(\mathrm{M}=\mathrm{Si}, \mathrm{Cu}, \mathrm{Nb}, \mathrm{Zr}, \mathrm{V}, \mathrm{Cr}, \mathrm{Mo}, \mathrm{Ta}, \mathrm{W} \ldots)$ deviennent, après un traitement thermique spécifique, nanostructurés. Ils sont constitués d'une phase nanocristalline (grains de taille $12 \mathrm{~nm}$ en moyenne) noyée dans une matrice amorphe (25-35\% du volume total). Du fait de la taille nanométrique des grains, l'anisotropie magnétocristalline est moyennée sur l'ensemble des grains. Il est alors possible, dans ce cas, d'induire par une action externe (champ magnétique ou contrainte mécanique) une anisotropie magnétique dans la direction souhaitée (Fig. 7). 

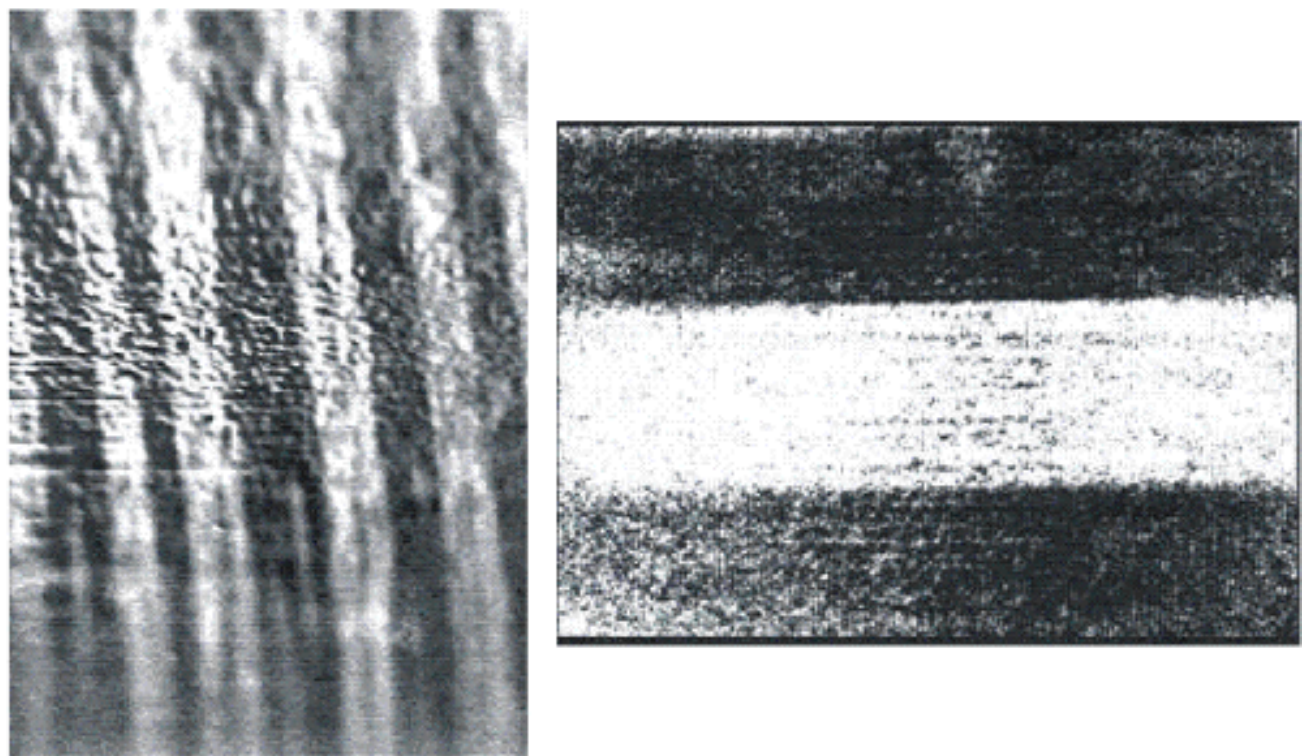

\section{$\longleftrightarrow$ Axe longitudinal du ruban $\Sigma$}

\section{$\mathrm{FeBSiCuNb}$}

\section{$\mathrm{FeBCuNbZr}$}

Fig. 7. Incidence sur les structures en domaines magnétiques de deux matériaux nanocristallins après recuit sous contrainte mécanique (100 MPa).

Certaines tôles fer-silicium de qualité HiB (haute induction) sont revêtues d'une couche qui exerce une contrainte de traction permanente sur la tôle. Il a été montré empiriquement que la contrainte affine les domaines (Fig. 8) et réduit les pertes. En conclusion, on peut compenser par effet magnéto-élastique un défaut d'orientation des grains, ce qui permet d'améliorer la qualité magnétique des tôles.
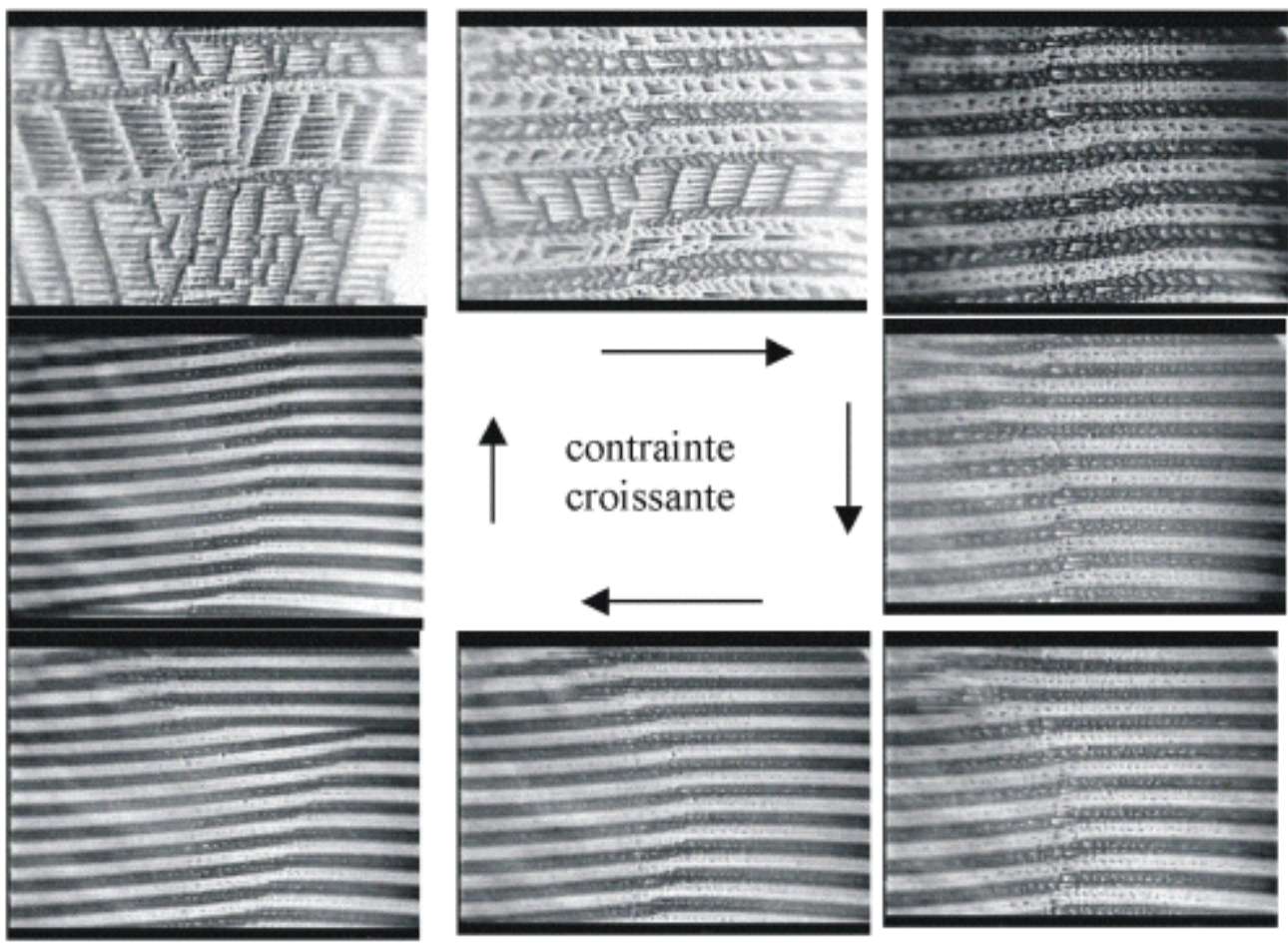

Fig. 8. Incidence d'une contrainte mécanique d'amplitude croissante sur une tôle fer silicium. 


\subsection{Influence de l'excitation magnétique (Hystérèse)}

De la structure en domaines (Fig. 9), on décrit les processus d'aimantation et donc la forme du cycle d'hystérèse et toutes les caractéristiques qui en découlent : perméabilité, induction rémanente, pertes fer...

Sur la figure 9 de gauche, on montre un processus d'aimantation par rotation de l'aimantation spontanée dans un alliage amorphe. Le champ magnétique extérieur est appliqué perpendiculairement aux domaines magnétiques.

Sur la figure 9 de droite, on montre un processus d'aimantation par déplacements des parois de Bloch. Le champ magnétique extérieur est appliqué parallèlement aux domaines magnétiques. Ci-dessous, on peut voir l'évolution de la structure en domaines pour différents états magnétiques.
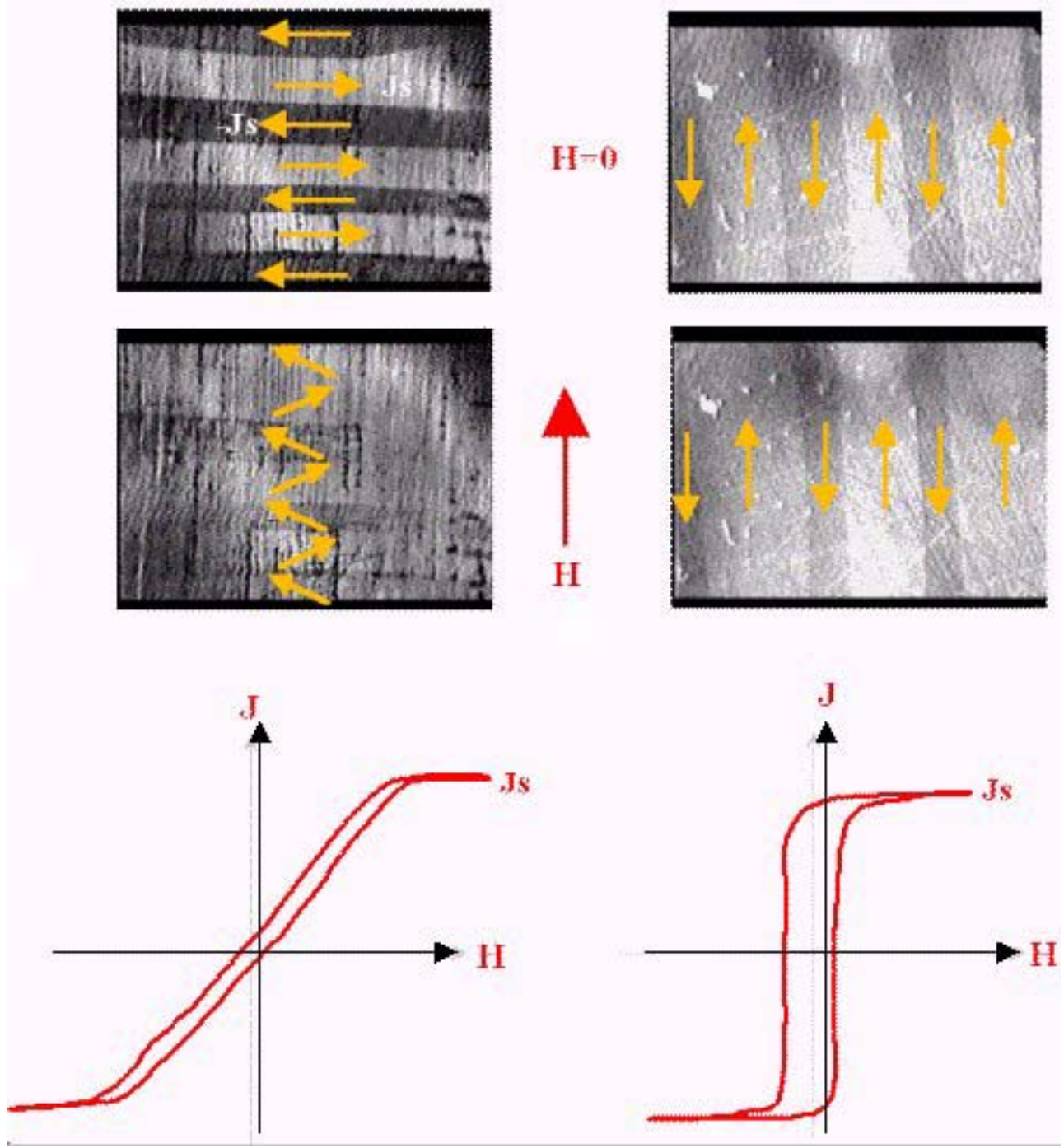

Fig. 9. Corrélation entre les structures en domaines magnétiques et le cycle d'hystérèse.

La plupart des étudiants ont du mal à se représenter physiquement l'hystérèse magnétique. Le diaporama cidessous (Fig. 10) montre un processus d'aimantation par déplacements des parois de Bloch. La vidéo numérique (Fig. 11), quant à elle, permet de mettre en évidence ce phénomène lié à la rotation des moments magnétiques sous l'influence d'un champ magnétique extérieur. 

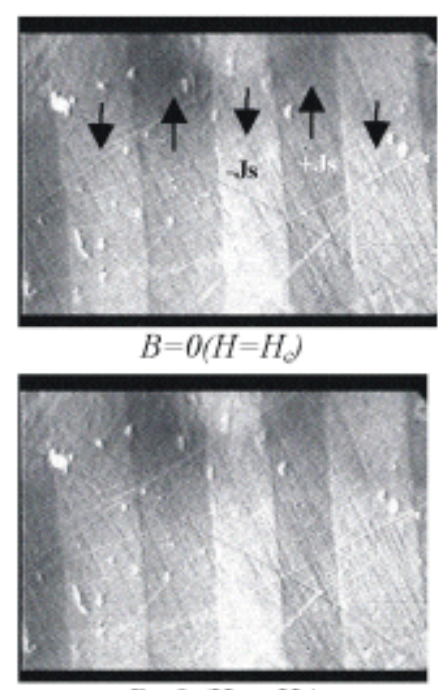

$B=0\left(H=-H_{\mathcal{D}}\right.$

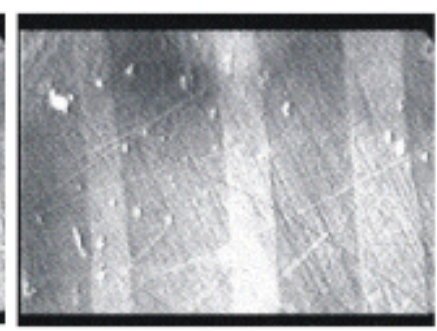

$B=B_{r}$

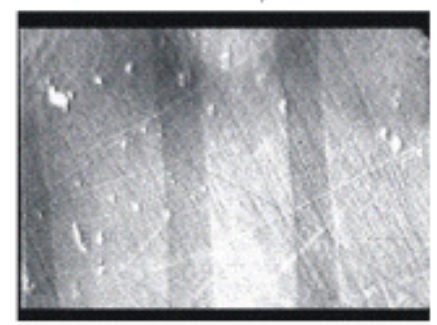

$B=-B_{r}$

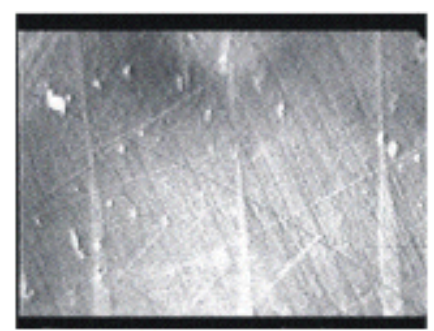

$B \approx B s a t$

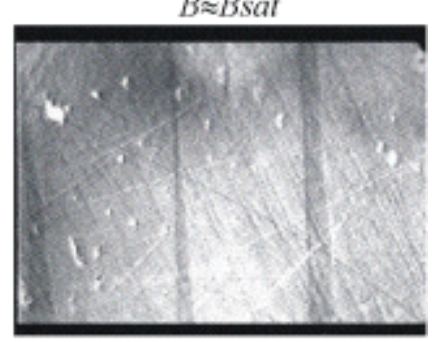

$B \approx-B s a t$

Fig. 10. Hystérésis microscopique par déplacements de parois de Bloch. Modification de la structure en domaines en fonction du champ magnétique appliqué.

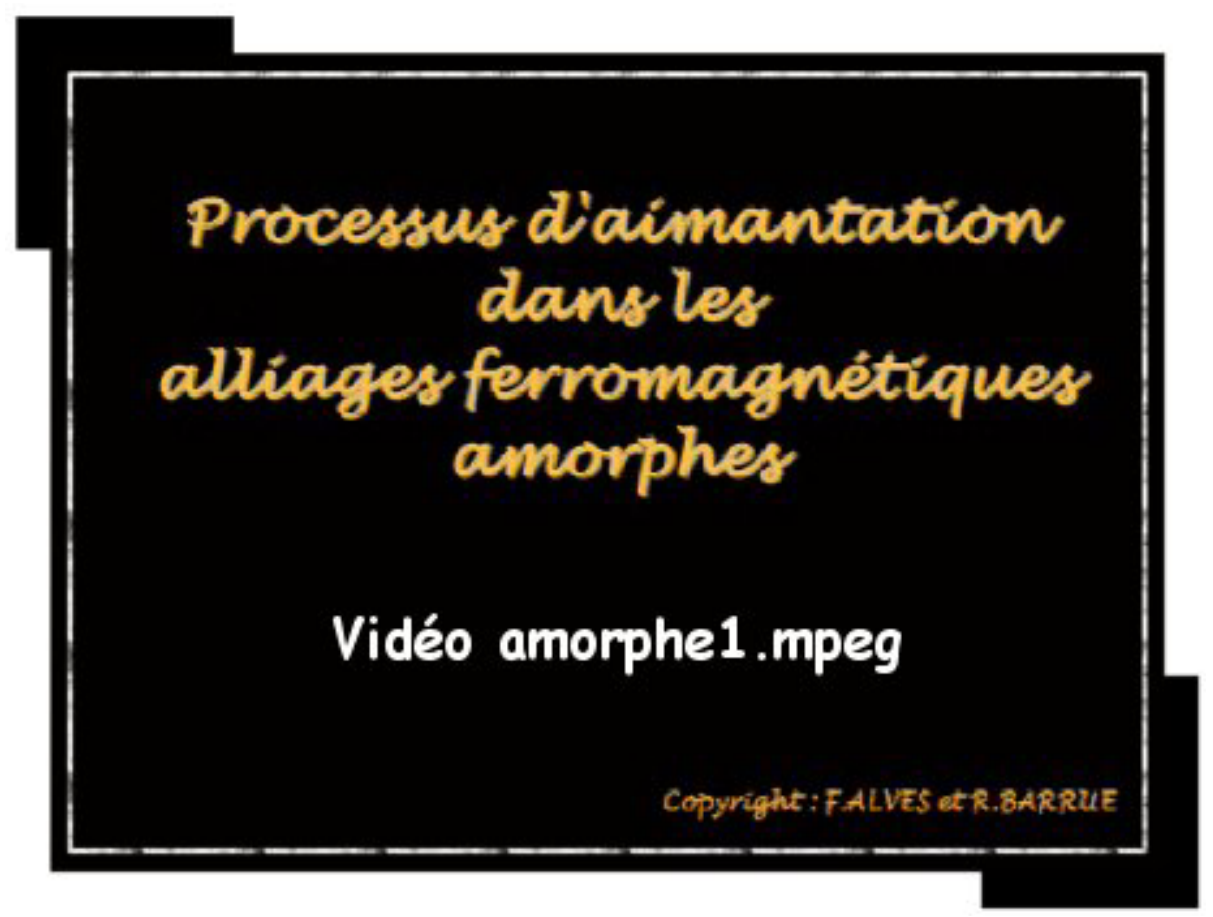

Fig. 11. Visualisation dynamique des processus d'aimantation dans les alliages ferromagnétiques amorphes (vidéo, fichier MPEG, 29 Mo). 


\section{Conclusion}

Une version plus complète de cet article et des images supplémentaires peuvent être obtenues dans le cédérom « Domaines Magnétiques » (Editions Micrelec) (Fig. 12).

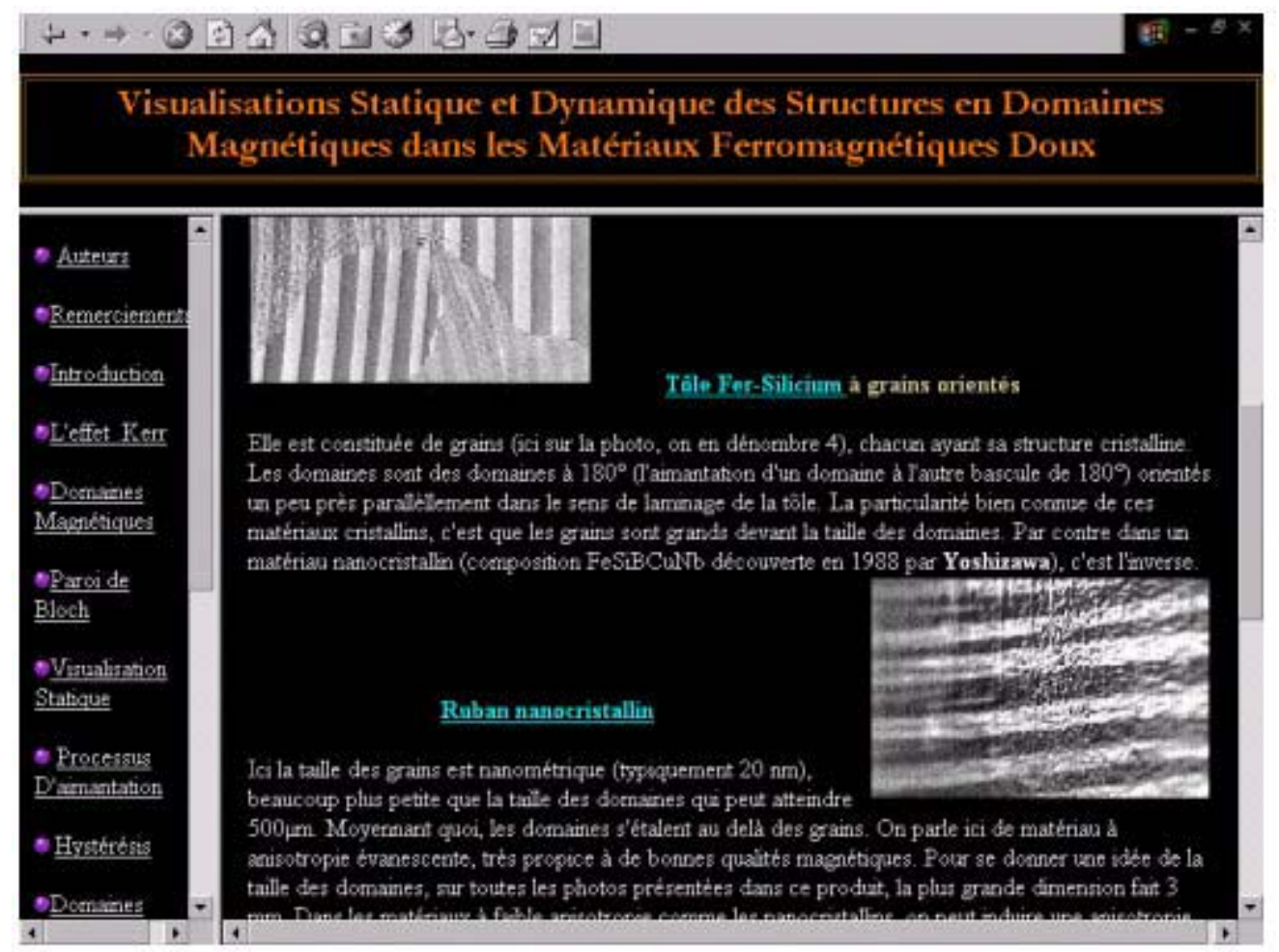

Fig. 12. Vue de la présentation du cédérom, aux éditions Micrelec.

Ce cédérom, inédit au niveau international, est consacré à l'observation des structures en domaines magnétiques. Véritable outil d'aide à la compréhension des phénomènes physiques relatifs au magnétisme à l'échelle des domaines, il permet, grâce à différentes séquences vidéo numérique originales, de contribuer à une approche plus pratique de l'enseignement de la physique restant trop souvent théorique. Présenté sous forme de didacticiel en pages HTML, le lecteur pourra naviguer au gré des différentes rubriques : état de l'art, explications sur le principe d'observation des domaines magnétiques par la méthode magnéto-optique de Kerr, notion de domaine magnétique et paroi de Bloch, visualisation de diverses structures en domaines rencontrées dans les matériaux cristallins, amorphes et nanocristallins, description des processus d'aimantation par rotations pures ou par déplacements de parois, observations du phénomène d'hystérèse local ou macroscopique, illustrations de l'effet magnéto-élastique, incidence de la fréquence sur la subdivision des domaines. Les diverses séquences présentées permettent d'avoir accès, par vidéo interposée, à un lourd équipement scientifique difficile d'approche à cause de l'optique (laser). Certains contenus ont déjà été présentés au niveau DEA et Agrégation de Génie Électrique, mais peuvent aussi être abordés au niveau du deuxième cycle moyennant certaines animations de vulgarisation.

\section{Bibliographie}

F. Alves et R. Barrué, Domaines électromagnétiques, CD-ROM pédagogique (Micrelec). 